\title{
Tracking affective components of satisfaction
}

\author{
Alexandra Coghlan, Philip Pearce
}

\begin{abstract}
This study seeks to heighten an appreciation of the multiple aspects of satisfaction by considering on-site travel experiences. Novel approaches to satisfaction are considered particularly appropriate in less structured and dynamic tourism settings where expectations are poorly defined and the expectancy disconfirmation paradigm is thus less applicable. Accordingly, this study examines the links between travel motivations, activities, emotions and satisfaction levels in tourists, using examples from a select number of tourists on dynamic volunteer tourism expeditions. Data were collected using diaries and analysed at individual and group levels, providing general experiential patterns and illustrating a linked approach to exploring tourists ' experiences, emotions and satisfaction. The results highlight that emotional variability occurs across time with distinct phases of positivity, annoyance and receptivity. This variability appeared to be linked to daily activities and personal characteristics. Furthermore, satisfaction levels did not always follow the patterns of emotional variability, which were in turn weakly related to expectations and motivations that were recorded at the start of the trip. Learning how to identify this variability may improve the monitoring and the management of the tourism experience and maximise tourists ' well being
\end{abstract}

\section{INTRODUCTION}

Satisfaction is an important and pervasive concept in tourism studies (Ryan, 1994). The assessment of visitor satisfaction is used at both a destination management level to monitor overall industry performance and at the scale of the individual business or organisation to explore and correct problems (Kozak and Rimmington, 1998; McCarthur, 2000; Kozac, 2001). At a more individual and psychological level, satisfaction matters to tourists themselves and numerous qualitative (and refl ective) accounts of tourist experience deal with the dimensions of visitor satisfaction (Noe, 1999 ; Small, 2003; Maoz, 2004). Finally, visitor satisfaction is often a goal of protected area management, and therefore important to government agencies as well as private enterprise (Griffin and Vacaflores, 2004).

The approach taken to satisfaction in this study is to view visitor satisfaction as an ongoing as well as a post-consumption attitude. In this approach, tourists can reflect immediately upon individual elements of an experience just after they have occurred as well as engaging in more delayed post-hoc evaluations in a longer time frame. Satisfaction in this sense is effectively an attitude in the full sense of the term embracing affective, cognitive and implicit behavioural elements (Pearce, 2005). It will be argued that this full attitudinal definition of satisfaction draws fresh attention to the affective dimension of satisfaction in particular, as opposed to more commonly used evaluations of specific, and management-chosen, facets of the tourism experience. The present 
wider definition prepares the way for a special focus on the elements of emotional responses to visitors , experiences and activities. This view is a partial corrective to the largely cognitive and rational appraisals of satisfaction employed in some of the more simplistic consumer behaviour work (Noe, 1999), as well as reflecting the importance of emotions in memory and event recollection. Ongoing research in this area of recollection provides evidence that experiences associated with emotional intensity are associated with greater memory confidence, but not always with high memory consistency (Levine and Pizarro, 2004).

It can also be suggested that the dominant style and timing involved in measuring satisfaction has become that of an attribute-based post experience survey (Millan and Esteban, 2004). There is an efficiency in this style of work as it enables large numbers of respondents to complete structured information at a mutually convenient time. It can also be argued that this approach has become so dominant in the tourism and business research world that satisfaction is now widely treated as a fixed outcome defined by the steps of Likert scales or similar response formats. The achievements of this style of satisfaction work are considerable, and numerous studies of satisfaction using this approach have been successfully conducted both to enhance tourism practice and develop conceptual models within tourism research (Mano and Oliver, 1993 ; Kozac, 2001).There are, however, several inadequacies in treating satisfaction as a single, fixed in time and invariant construct. A consideration of the existing and recent literature in the following section highlights some of these concerns and some alternate views and measures of satisfaction will be the central concerns of the present research.

In a broad sense this study seeks to heighten an appreciation of the multiple aspects of satisfaction by focussing particularly on assessing satisfaction during or closely linked to tourists ' on-site travel experiences. This is an attempt to close the gap between assessing satisfaction and the immediate embodied visitor experience (cf. Tribe, 2004). The full rationale for investigating satisfaction close to the tourists ' on-site experience and in emphasising its emotional components derives from the achievements of a few select pioneering studies as well as from recent emphases on tourist activity research (Bowen, 2002 ; Beedie, 2003). The context and research setting for the present work lies in assessing the experiences of volunteer tourists. Volunteer tourism has been used as a backdrop for this research as it can involve novel and somewhat unpredictable travel expeditions that generate vivid, enduring ' fl ashbulb memories ’ (Brown and Kulik, 1977).

\section{LITERATURE REVIEW}

In the field of consumption experiences, satisfaction and emotions are inextricably linked (Westbrook, 1987 ; Oliver, 1993). For instance, Zins (2002, p. 4) argues that there is ' ample evidence that emotional reactions associated with the consumption experience are fundamental for the determination of satisfaction '. Satisfaction researchers are increasingly recognising the need to incorporate both affective and cognitive components in modelling consumer satisfaction (Bigne et al, 2005). This is particularly important in tourism services where emotional involvement appears to play an important role in the tourist experience and a focus on the tourists ' subjective experiences highlights the need to integrate cognitive and emotional concepts in order to explain tourist satisfaction (Zins, 2002 ; Bigne et al, 2005). Thus, tourism experiences are one form of consumption experience, and emotions elicited by tourism experiences may be studied using consumption experience models. 


\section{Real time satisfaction measures}

Over the past decade or so, authors have supported the notion that on-site experiences deserve greater attention and should be considered an integral component of tourist satisfaction (Arnould and Price, 1993 ; Webb, 2002 ; Chhetri et al, 2004). Real-time satisfaction measurements are an appraisal of one 's current or very recently experienced state, including current cognitions and emotions. This is an important point as it relates to the preceding paragraphs on the need to integrate measures of emotion into satisfaction studies. Furthermore, unlike the traditional attribute-based post experience survey that depends upon a cognitive evaluation of those attributes, real-time measures are not so directly affected by issues such as recall, changed contexts, hindsight bias or positivity (Bowen, 2002). In particular, real-time satisfaction measurements allow for fl uctuations and variability in satisfaction levels and cognitive and affective states (Hull et al, 1992). Such measurements are also suited to answer questions such as what kinds of direct environmental attributes influence positive and / or negative feelings; how does the social setting relate to individual experiences; or how does the changing quality of service influence satisfaction? (Hull et al, 1992 ; Stewart and Hull, 1996).

Although other satisfaction measures are used in tourism studies, the most commonly cited ones being expectancy-disconfi rmation paradigm and servqual measures, it has been suggested that on-site measurements are particularly appropriate where tourists are unlikely to have well-defined expectations. In these cases, measures based on the expectancy disconfirmation paradigm are unlikely to provide an adequate measure of satisfaction. Settings where researchers have used real time measurements include hiking trips, river rafting, small group, long-haul, soft-adventure tours and adventure tourism in general (Arnould and Price, 1993 ; Weber, 2001 ; Bowen, 2002 ; Beedie, 2003 ; Chhetri et al, 2004). Real time experience measures in these cases can cover a wide range of meanings including mood, emotions and feelings of individual tourists (Chhetri et al, 2004). According to most of these authors, investigating a tourist ' $s$ on-site experience and emotions is a useful, and perhaps essential, step in creating a broader understanding of visitor satisfaction.

\section{The role of emotion in satisfaction}

The present study takes the approach that affect is an antecedent to satisfaction and that satisfaction results from evaluating the affect derived from a consumption experience. Forgas (1995) describes the ways in which affect may infl uence satisfaction through affect infusion (or affect transfer), suggesting that positive emotions influence judgements by shaping what it noticed, encoded and retrieved in the judgement process. lternatively, individuals may use their affective state as a shortcut to infer their overall attitude to an object.

Being able to identify an individual's emotions at a given time becomes very important in either case, and being able to measure evolving stages of emotions throughout the course of an experience is equally important (Dube and Morgan, 1998). In many studies, measures of satisfaction are built upon multi-dimensional scaling approaches to the study of emotions. Such studies identify the underlying dimensions of emotions. The first of these dimensions is pleasantness - unpleasantness, identified as far back as 1897 by Wundt (cited in Russell et 
al, 1989). The second dimension that occurs with a high degree of regularity in multi-dimensional scaling studies is the arousal or level of activation dimension.

Perhaps one of the best known models to have arisen out of the two dimensional scaling of emotions is Russell ' s circumplex model, in which he proposed that affective states are best represented as a circle in a two dimensional space (Figure 1). He labelled the fi rst dimension (on the horizontal axis) pleasure - displeasure and the second dimension (on the vertical axis) arousal - sleep. The eight affect concepts that he used in the multi-dimensional scaling procedure could then be said to fall at precise points along the axes in a circular order. Furthermore, Russell suggested that this circular ordering of the eight concepts may be because of the fuzziness of the boundaries of affect words, for example, the boundaries of pleasure and excitement overlap considerably, placing them next to each other in the circle.

Based upon these measures of affect developed in the field of psychology, other researchers have been able to develop and adapt scales to be used in the study of consumption emotion. Richins (1997) argues that consumption emotions differ in character and intensity from emotions that are experienced in other contexts. She therefore set out to generate a list of affective states that would capture the full range of consumption emotions. The resulting multi-dimensional emotion space revealed a flattened circumplex, with a greater differentiation of emotions on the fi rst dimension than on the second (Figure 2). The dimensions that describe these emotional states are similar to those of Russell, but the space contains more descriptions and is arranged in a mirror image (that is inverted and reversed) format.

Generally, research into emotions undertaken in the field of tourism and leisure research have been based upon such multi-dimensional appraisals of emotional space. Hull et al (1992) adapted Russell and Mehrabian 's Pleasure, Arousal, Dominance scales to include four dimensions of affect, namely bored, excited, rushed and relaxed, in their study of experience patterns of hikers in Colorado. More recently, Zins (2002) attempted to link consumption emotions, experience quality and satisfactions using the positive affect, negative affect scale (PANAS) and the circumplex scale. The present research builds upon these earlier studies in an attempt to understand tourist satisfaction based upon a multi-component approach to measuring emotion.

This study tracks the affect-based components of satisfaction in a setting (volunteer tourism) that provides opportunities to experience the range of emotions presented in Figure 2 . The principle objective of this work is to present an alternative method of tracking tourists ' on-site experiences and satisfaction levels. The approach represents a departure from studies of visitor satisfaction, which use a post-trip survey to rate tourist satisfaction with a range of attribute-based items (see Millan and Esteban, 2004 , for a review of this approach). Instead the work is based upon continuous, daily, multi-faceted measurements of satisfaction and affect. 


\section{AIMS AND OBJECTIVES}

The work presented here is exploratory in nature, and so long as the role of emotions in satisfaction is still being debated by psychologists, no preconceptions of the nature of the relationship between emotions and satisfaction were used to guide hypothesis testing. Additionally, a record of pre-trip motivations permit some links among affect, satisfaction, and expectations and motivations to be partly explored, providing a link with the body of tourism work on expectancy (dis)confirmation models in satisfaction ratings. The principal aims of this study are therefore twofold:

1. To illustrate a new style of analysing tourism experiences, emotions and satisfaction by applying a modified circumplex model of affect to a daily analysis of on-site experiences.

2. To develop a linked approach to explore how motivations and activities infl uence tourists , experiences, emotions and satisfaction.

\section{METHOD}

In order to investigate the links between affective experiences of tourists and their reported satisfaction, daily diaries were used to record overall affect levels, respondents ' activities and their feelings with regard to those activities. Daily diaries provide a rich source of information on the structured, time dependent, on-site experiences of the volunteer tourist, and are well established, although not yet common within tourism research (cf. D. Pearce, 1988 ; Phillimore and Goodson, 2004). Some examples of authors who have used the diary technique include Pearce (1981) and Fennell (1996), who both used some form of reporting daily or hourly events and respondent's affective states in their studies to assess changes in moods throughout the holiday experience.

Thirty volunteer diaries were distributed to a volunteer tourism organisation who had agreed to participate in this study. This particular organisation ran live-aboard dolphin research expedition, where groups of up to eight volunteers and three staff spent a maximum of 9 days (weather permitting) at sea collecting data on the distribution and behaviour of various dolphin species in the Mediterranean. The choice of this type of setting was deliberately intended to obtain a sequence of data in an unpredictable tourism setting. At the start of each expedition, all the tourists were given a briefi ng concerning the research, its aims and what would be required of them should they choose to participate in the study. The diaries consisted of several distinct sections, the fi rst of which asked the respondents to describe the activities that they had undertaken that day. The final section concerned the affective state of the volunteers that day, as well as their on-site, real time satisfaction levels. The methodology used to investigate each of these three aspects of the volunteers ' experiences is described in more detail below. 


\section{Sample}

A total of 16 volunteers from three different expeditions completed the surveys (53 per cent response rate). The tourists were predominantly over the age of 50. They were North American or British, with a high level of previous travel experience, some volunteer tourism experience and conservation involvement through donations to charities.

\section{Tourist motivations and expectations}

The motivations and expectations of this group of volunteer tourists were examined by providing checklists of motivational items tailored to the setting. This checklist was derived from past studies on tourist and recreation motivations (Manfredo et al, 1996 ; Pearce and Lee, 2005), volunteering (Bonjean et al, 1994) and volunteer tourism (Weiler and Richins, 1995 ; Galley and Clifton, 2004). The list was then presented to actual volunteer tourists and volunteer tourism project leaders for comment and feedback, before developing the final data collection tool. The data were collected in the diaries using a combination of open questions and a Likert-scale survey based upon previous studies of tourist and volunteer tourist motivations (Pearce and Lee, 2005). The actual motivations of the volunteer tourists will not be examined empirically in depth here, but used principally as illustrative material in reporting individual cases. Instead, the tracking of activities, emotion and satisfaction will form the focus of this study using an exploratory approach and acknowledging that the sample sizes are small.

\section{Tourists ' assessment of daily activities}

Descriptions of activities were split into those activities that took place in the morning, afternoon and evening. Respondents were therefore asked to complete this section at three points during the day, such as lunchtime, after dinner and before going to bed. Volunteers were asked to describe each activity as succinctly as possible and to note the time of the activity as well as any comments they might have about the activity. This procedure follows a similar procedure set out by Pearce (1981) and Fennell (1996) .

\section{Daily affective states}

On the third and final page of the daily diary, volunteers were asked to record their daily affective states. They recorded this on a modified version of the circumplex model of affect developed for the analysis of emotions in consumption experiences (Russell, 1980 ; Richins, 1997 ; Zins, 2002). The emotions cited by Richins that were believed to be irrelevant to volunteer tourism experiences, for example, disgusted, ashamed, tender, were excluded from this study. The remaining emotions were presented in a circle, and volunteers were asked to tick which emotions they had experienced that day. Emotions that were identified as relatively similar in previous studies were presented in adjacent segments of the circle. Furthermore, respondents were asked to indicate the intensity level of the emotion that they had experienced, by placing their tick in the centre (low level), middle 
(medium level) or on the edge of the circle (high level) (Figure 3). Again, this allowed the type and intensity of affective states to be analysed both at an individual level and within and between expeditions. Volunteer tourists were also provided with the opportunity to add written comments concerning their feeling states after they had completed the wheel.

\section{Satisfaction levels}

Finally, the respondents were asked to rate their answer to the following question ' How satisfied are you with your experience right now? ' on a Likert scale of $1-5$, where 1 equals not at all, and 5 equals very much. The data collected were used to determine overall satisfaction with the expedition on a daily basis as well as to detect changes in satisfaction throughout the trip and among respondents.

\section{RESULTS}

It is important to be clear on the nature of the results from this study. The data are presented at a total or combined level for the volunteer tourists $(\mathrm{N}=16)$ for three separate expeditions all with the one volunteer tourism company.

\section{The volunteers' on-site emotions}

The volunteers were generally more positive than negative in their emotions. Figure 4 shows that emotions such as happy, contented, optimistic and pleased are frequently recorded at high levels, whereas emotions such as worried, frustrated, tense, lonely, unfulfilled, discontented, irritated, sad and depressed do occur among some volunteers, but generally at lower levels.

By dividing the emotion wheel into three segments - a broadly positive segment, a smaller annoyance segment and a negativity segment - a broad picture of these relationships can be conveyed. The emotion scores are predominantly in the positivity sector and the overall satisfaction scores are generally positive and favourable (4.2 or higher on the 5-point scale).

Trends of fluctuating satisfaction and emotion types are noted throughout the week. Day 5 included the most negativity scores, including high level of frustration, medium levels of irritation and lack of fulfilment, and low level of feeling discontented, worried and tense.

This is also the day with the lowest overall satisfaction score. Day 5, with the most negativity scores, is the day with the lowest overall satisfaction score. Days 4, 6, 8 and 10, where some frustration linked emotions are identified, vary in the satisfaction scores reported but they are generally lower scores. It is important to recognise, however, that these kind of data and their interpretation rests on the cut-off points used to decide 
between high, intermediate and low counts of the affective responses.

The overall relationship between these emotion wheels and the simple one-item appraisals of daily satisfaction are presented in Table 1 . To simplify the measures of emotion presented in this table, we returned to Richins ' model (Figure 2) and measured the distances between emotions as they fell on the two dimensions. Where the distances were greater than average, that is, between the emotions lonely and calm $(4 \mathrm{~cm})$, excited and frustrated $(10.3 \mathrm{~cm})$ and discontented and worried $(3.5 \mathrm{~cm})$, the circle was divided into segments to represent groups of spatially close emotions. The segments broadly represent positive emotions such as happiness, negative emotions such as sadness and emotions that represent annoyance.

A further examination of the patterns of data revealed in Table 1 displays some observable consistencies. In providing an overall appraisal of respondent 's emotions, Day 1 shows a good deal of emotional variability, but there are strong areas of positivity. Days 2 and 3 are similar and are among the most positive registrations of emotion for the entire sequence of days. Days 4, 5, 6 and 7 are highly consistent; they are slightly less positive than days 2 and 3 and some tendencies towards annoyance are expressed. Day 8 shows the maximum amount of annoyance with the largest number of emotions of any day in the negativity and annoyance quadrant. Nevertheless day 8 is still full of some positive emotions, thus registering the bipolar complexity of the information. Days 9 and 10, the final two days of the expedition, show more receptivity (or passive emotional states) and less intense positive emotions.

\section{Linking tourist motivations and activities with emotions and satisfaction}

The second aim of this article is to illustrate a novel method of linking tourist motivations and activities with emotions and satisfaction. Motivations in this case are used to assess, at a crude level, elements of expectations and the need fulfilment aspect of satisfaction and expectation (dis)confirmation models. The respondents , motivations that were noted upon arrival (before the expedition briefing) are matched up with the daily activities that are reported in each respondent 's diary, and, where matches were noted; this is reported as a tick in Tables $2-5$. To illustrate these links, four examples have been drawn out of the sample of 16 respondents. These four examples using the same data provided above serve to demonstrate how all of these elements can be linked together to build a more comprehensive and, in this instance, a case-by-case understanding of the tourism experience. A closer inspection of each example will reveal slightly different perspectives on the respondents , experiences (Tables $2-5$ ).

The first respondent reported that her experience focussed strongly on all the new things she experienced, and she enjoyed any new and challenging activities (Table 2). However, she often had her ' down time' , where she reports feeling mellow, and uses terms such as uneventful to describe the day. An example of a diary entry on these days is ' it was nice to have some time off, but I would have rather been out on the water, at least there we have jobs to do and don ' $t$ have to spend all that much time together'. On these days her mood changed from 
encouraged, happy, pleased and excited to depressed, lonely and sad. Her satisfaction levels, on the other hand, did not vary during the course of the trip and stayed high throughout. A comparison of the daily activities that she reported in her diary and travel motivations she cited before the trip commenced overlap to a large extent, perhaps indicating an adequate match between destination image and on-site experience.

The second respondent recorded her most positive emotions and highest satisfaction on days when she saw the dolphins and watched their behaviour (Table 3). Other positive elements for her were the crew, whom she found to be very positive and helpful, being out at sea, the scenery and good food. On the other hand, she did mention certain factors that detracted from her experience, such as the poor weather conditions, days when no sightings were made or the prioritisation of the turtle research over the dolphin research. On these days, she recorded some negative emotions such as feeling highly discontented, unfulfilled, frustrated and sad, and her satisfaction scores dropped to ' somewhat satisfi ed'.

The third respondent took a few days to settle into the expedition (Table 4). On day 3 she mentions feeling physically and emotionally tired, and on day 4 she feels overly sensitive to the crew's reactions to her shortcomings. She says that she wants ' not necessarily the crew's approval, but not their disapproval '. By the third day, however, she appears to be feeling more relaxed and comfortable with the expedition and negative comments about others have disappeared from her diary. She does remain highly introspective throughout the rest of the trip, however, never quite feeling completely at ease, while being thrilled by the dolphin sightings, enjoying getting to know the other team members, learning about the project and the research techniques and viewing the animals themselves.

The fourth respondent's emotions and satisfaction levels also develop with time (Table 5). She starts the trip feeling anxious, and struggles to find a balance with the rest of the group. She mentions that she is annoyed that other members of the team put in less effort and appear to care less about the chores than her. On day 3 she reports ' I feel extremely disappointed that the thing I was assigned to do, I will not get a chance to experience in this trip '. She is also feeling tired, having slept poorly for the first few days. After this difficult day her satisfaction scores and moods started to improve. She describes the days with sightings as brilliant, where times flies by and everything else is forgotten, so that despite the hard work it is a thrill for all.

\section{DISCUSSION}

The first aim of this study was to develop and illustrate a new style of analysing tourism experiences, emotions and satisfaction whereas the second study aim was constructed to investigate the links among these outcome factors. In an important sense the data collected in this study of volunteer tourists are used more as an illustration of the potential of the methods employed, rather than serving as a comprehensive study of the volunteer tourists. 
A substantial finding to note, and one connected to the second study aim, is that the information reported in the sequence of emotion wheels shows little correspondence with the overall daily satisfaction mean scores as reported in Table 1 . Furthermore, an important and potentially insightful feature of Tables $2-5$, and the descriptions of satisfaction provided to accompany these tables, is the limited relationships among these measures. It would appear, in particular, that the more generic global satisfaction measures only partially pick up the emotional variability revealed in the circumplex style assessments used here. For example, respondent 3 who records some negative emotions in her day-to-day emotional wheels (a perspective summarised overall in Table 5 in the annoyance row) does record one satisfaction score of 3 on day 5, but in general shows the kind of positivity bias and skewed satisfaction results reported in many assessments in tourism (cf. Ryan, 1994). By way of contrast the overall emotion wheel of respondent 2 is markedly more concerned with negative emotional remarks, but the direct satisfaction scores (when provided) are only slightly lower than respondent 3 . The central implication here is that the emotion wheels are more searching descriptors of the tourists' experiences and offer a discerning view of the sequence of visitors ' perceived well being during travel experiences. This fi nding has important consequences for managers interested in understanding evaluations of on-site satisfaction and consumption emotions. The results suggest that the relationship between affect and satisfaction is not a simple one in tourism, and that other factors, perhaps cognitive evaluations of current state, come into play. The mismatch between reported satisfaction (good) and emotional states (variable) may help to explain some of the diffi culties that are encountered when organisations seek to engage further volunteer tourism support after the conclusion of their on-site involvement. The patterns of emotional fl uctuation that are described above are a combined product of the individual fluctuations portrayed in part in Tables $2-5$. The material presented in Tables $2-5$ begins to address the ways in which the emotion wheels can be used to link the study of motivations, activities and satisfaction and represents a more empirical representation and extension of emotion-linked assessments in qualitative research accounts where highly detailed descriptions of travel experience are often presented (cf. Phillimore and Goodson, 2004).

Another key outcome of the work, and this implication relates more directly to the fi rst aim of the research, is that these kinds of time-based and sequential records of tourists ' self-reported emotional states may be considered as applicable in a number of tourism contexts and for a number of purposes. This type of data is relatively simple to collect, as the tourists were eager to maintain a diary-style record of their experiences. Additionally, the diaries were easily formatted to collect this information in a manner that can be readily analysed. This may be particularly relevant for those tourism sectors that seek to track the commitment levels of their participants such as those involved in conservation activities and public good tourism. In a more commercial sense too, there are few studies of how emotional and satisfaction trajectories are played out when tourists stay with the one business (such as a tour company or at a resort). Results from the daily emotion plots discussed in this work may provide novel information for these business managers. 
One way to assess all the information reported here is from the perspective of the tour organiser. Consistent group-based information of the type described in Table 1 can lead to action on the part of, say, the volunteer tourism company. It may be possible to recognise and identify common ' danger days' in the sequence of visitor emotions with their experience. Similar phases of adjustment and adaptation in a wide range of human contact and social groups are an established part of the culture contact and tourism literature (cf. Ward et al, 2001). The pattern identified here is less important than the prospect of identifying such patterns. Additionally, an assessment of critical incidents may assist managers to re-focus the energy of the group for superior outcomes (cf. Pritchard and Havitz, 2006). The managerial response to the shifting emotional patterns of volunteer tourism is only one remedial action, as the tourists themselves can prepare for periods of difficulty if some pre-departure information on the complexities and dynamics of being in these kinds of work-leisure groups can be provided. Importantly, the atmosphere and group dynamics that influence the experience of volunteer tourist groups extend to many other kinds of tourist settings. Such group-based affective responses measured over a set time period are applicable, among others, to adventure tour expeditions, group coach travel and resort-based tourism.

In striving to improve our understanding of visitor experiences in continuing tourist experiences, the initial work conducted in this study can be viewed as supporting the value of and suggesting the need for attention to affective components. The small sample sizes and the case study approach presented in the reporting of this research require future researchers to explore the complexities of using the tool with more respondents and in other contexts. In particular, issues of the reliability and validity of these measures have not yet been thoroughly examined, and should be investigated in detail. The emotion wheels trialled in this study may need simplification for widespread tourism business use (for example, by using fewer descriptors in the positivity / negativity / annoyance / receptivity segments). The wheels may also provide the more detailed kind of feedback on customer responses that aids industry practice and promotes the application of tourism research. In a broad sense, and observing these caveats about appropriate checking and verification procedures, the work represented here outlines a promising data collection tool that allows for detailed and rapid assessments of on-site emotions, activities and satisfaction levels. 


\section{REFERENCES}

Arnould , E . J . and Price, L . L . (1993) River magic: Extraordinary experience and the extended service encounter . Journal of Consumer Research 20 : $24-45$.

Beedie , P . (2003) Mountain guiding and adventure tourism: Reflections on the choreography of the experience . Leisure Studies 22: $147-167$.

Bigne , J . E . , Andreu , L . and Gnoth , J . (2005) The theme park experience: An analysis of pleasure arousal and satisfaction . Tourism Management 26 : 833 - 844 . Bonjean, C.M ., Markmam, W.T . and Macken ,

P.O. (1994) Measuring self-expression in volunteer organisations: A theory-based questionnaire . Journal of Applied Behavioural Science 30 : $487-515$.

Bowen , D . (2002) Research through participant observation in tourism: A creative solution to the measurement of consumer satisfaction/ dissatisfaction (CS/D) among tourists . Journal of Travel Research $41: 4-14$.

Brown, R . and Kulik, J . (1977) Flashbulb memories . Cognition 5 : 73 - 99 .

Chhetri , P ., Arrowsmith , C . and Jackson , M . (2004) Determining hiking experiences in nature-based tourist destinations . Tourism Management 25 (1) : $31-43$.

Dube , L . and Morgan , M . S . (1998) Capturing the dynamics of in-process consumption emotions and satisfaction in extended service transactions . International Journal of Research in Marketing 15 : $309-320$

Fennell , D . A . (1996) A tourist space-time budget in the Shetland Islands . Annals of Tourism Research $23(4)$ : $811-829$.

Forgas , J.P . (1995) Mood and judgement: The affect infusion model (Aim) . Psychological Bulletin 11 : $39-66$.

Galley , G . and Clifton , J . (2004) The motivational and demographic characteristics of research ecotourists: Operation Wallacea volunteers in South East Sulawesi, Indonesia . Journal of Ecotourism 3 : $69-82$.

Griffin , T . and Vacaflores, M . (2004) A Natural Partnership: Making National Parks a Tourism Priority. Gold Coast, Australia: Sustainable Tourism CRC .

Hull IV , R . B ., Stewart, W . P . and Young, K . Y . (1992) Experience patterns: Capturing the dynamic nature of a recreation experience . Journal of Leisure Research 24 (3) : 240 - 252 .

Kozac , M . (2001) A critical review of approaches to measure satisfaction with tourist destinations . In: J.A. Mazanec, G. Crouch, J.R. Brent Ritchie and A. Woodside (eds.) Consumer Psychology of Tourism Hospitality and Leisure, Vol. 2 Wallingford, CT and Oxon: CABI Publishing, pp. $303-320$.

Kozak , M . and Rimmington , M . (1998) Benchmarking: Destination attractiveness and small hospitality business performance . International Journal of Contemporary Hospitality Management. Wallingford 10 (5) : $74-78$. 
Levine , L . J . and Pizarro , D . A . (2004) Emotional and memory: A grumpy overview . Social Cognition 22 (5) : $530-554$.

Manfredo , M . J . , Driver , B . L . and Tarrant, M . A . (1996) Measuring leisure motivation: A meta analysis of the recreation experience preference scales . Journal of Leisure Research 28 : $188-213$.

Mano , H . and Oliver , R . L . (1993) Assessing the dimensionality and structure of the consumption experience: Evaluation scaling and satisfaction . Journal of Consumer Research 20 : $451-466$.

Maoz , D . (2004) The conquerors and the settlers: Two groups of young Israeli backpackers in India . In G. Richards and J. Wilson (eds.) The Global Nomad. Clevedon, UK: Channel View , pp. 109 - 122 .

McCarthur , S . (2000) Beyond carrying capacity: Introducing a model to monitor and manage visitor activity in forests . In X. Font and J. Tribe (eds.) Forest Tourism and Recreation: Case Studies in Environmental Management. Wallingford, CT: CABI Publishing, pp. 259 - 278 .

Millan , A . and Esteban , A . (2004) Development of a multi-item scale for measuring customer satisfaction in travel agencies services . Tourism Management 25 (5) : 533 - 546 .

Noe , F . P . (1999) Tourism Service Satisfaction. Champaign, IL: Sagamore .

Oliver , R . L . (1993) Cognitive, affective and attribute bases of the satisfaction response . Journal of Consumer Research 20 : $418-430$.

Pearce, D . (1988) Tourist time-budgets . Annals of Tourism Research 15 : $106-121$.

Pearce, P . (1981) Environmental shock: A study of tourists' reactions to two tropical Islands . Journal of Applied Social Psychology 11 (3) : $268-280$.

Pearce, P . L . (2005) Tourist Behaviour. Clevedon, UK: Channel View Publications .

Pearce , P . L . and Lee , U . -I . (2005) Developing the travel career approach to tourist motivation . Journal of Travel Research 43 : $226-237$.

Phillimore , J . and Goodson , L . (eds.) (2004) Qualitative Research in Tourism. London: Routledge .

Pritchard , M . and Havitz , M . (2006) Destination appraisal. An analysis of critical incidents . Annals of Tourism Research 33 (1) : $25-46$.

Richins , M . L . (1997) Measuring emotions in the consumption experience . Journal of Consumer Research 24 (2) : $127-146$.

Russell , J . (1980) A circumplex model of affect . Journal of Personality and Social Psychology 39 (6) : $1161-1178$.

Russell , J . A ., Weiss, A . and Mendelsohn, G . A . (1989) Affect grid: A single-item scale of pleasure and arousal. Journal of Personality and Social Psychology 57 (3) : 493 - 502 .

Ryan , C . (1994) Leisure and tourism - The application of leisure concepts to tourist behaviour - A proposed model . In: A.V. Seaton (ed.) Tourism: The State of the Art. Chichester, UK: John Wiley, pp. $294-307$.

Small , J . (2003) Voices of older women tourists . Tourism Recreation Research 28 (2) : 31 - 39 .

Stewart , W . P . and Hull IV , R . B . (1996) Capturing the moment: Concerns of the in situ leisure research . Journal of Travel and Tourism Marketing $5(1 / 2): 3-20$. 
Tribe , J . (2004) Knowing about tourism: Epistemological issues . In J. Phillimore and L. Goodson (eds.) Qualitative Research in Tourism. London: Routledge , pp. 46-62 .

Ward , C ., Bochner , S . and Furnham , A . (2001) The Psychology of Culture Shock, 2nd edn. East Sussex, UK: Routledge .

Webb , D . (2002) Investigating the structure of visitor experiences in the little sandy desert, Western Australia . Journal of Ecotourism 1 (2 and 3) : $149-161$.

Weber , K . (2001) Outdoor adventure tourism: A review of research approaches . Annals of Tourism Research 28 (2) : $360-377$.

Weiler , B . and Richins , H . (1995) Extreme, extravagant and elite: A profile of ecotourists on Earthwatch expeditions . Tourism Recreation Research 20: $29-36$.

Westbrook, R . A . (1987) Product/consumptionbased affective responses and post-purchase processes . Journal of Marketing Research 24 : 258 - 270 .

Zins , A . H . (2002) Consumption emotions, experience quality and satisfaction: A structural analysis for complainers and non-complainers . Journal of Travel and Tourism Marketing 12 (2) : $3-18$. 


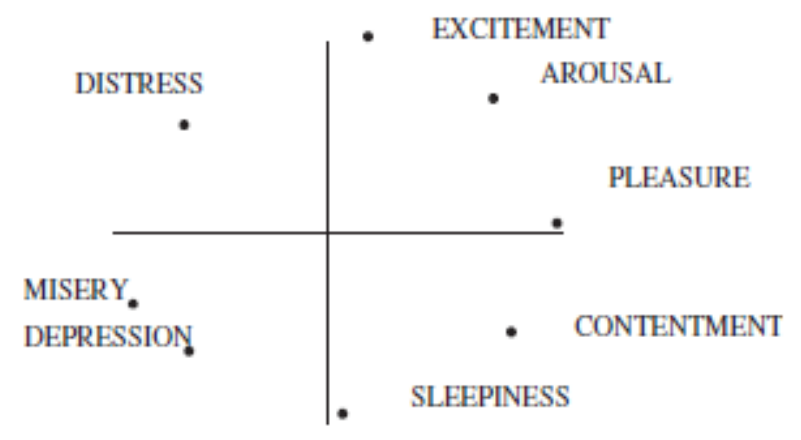

Dimension one:pleasure-displeasure

Figure 1 : The circumplex model after Russell (1980)

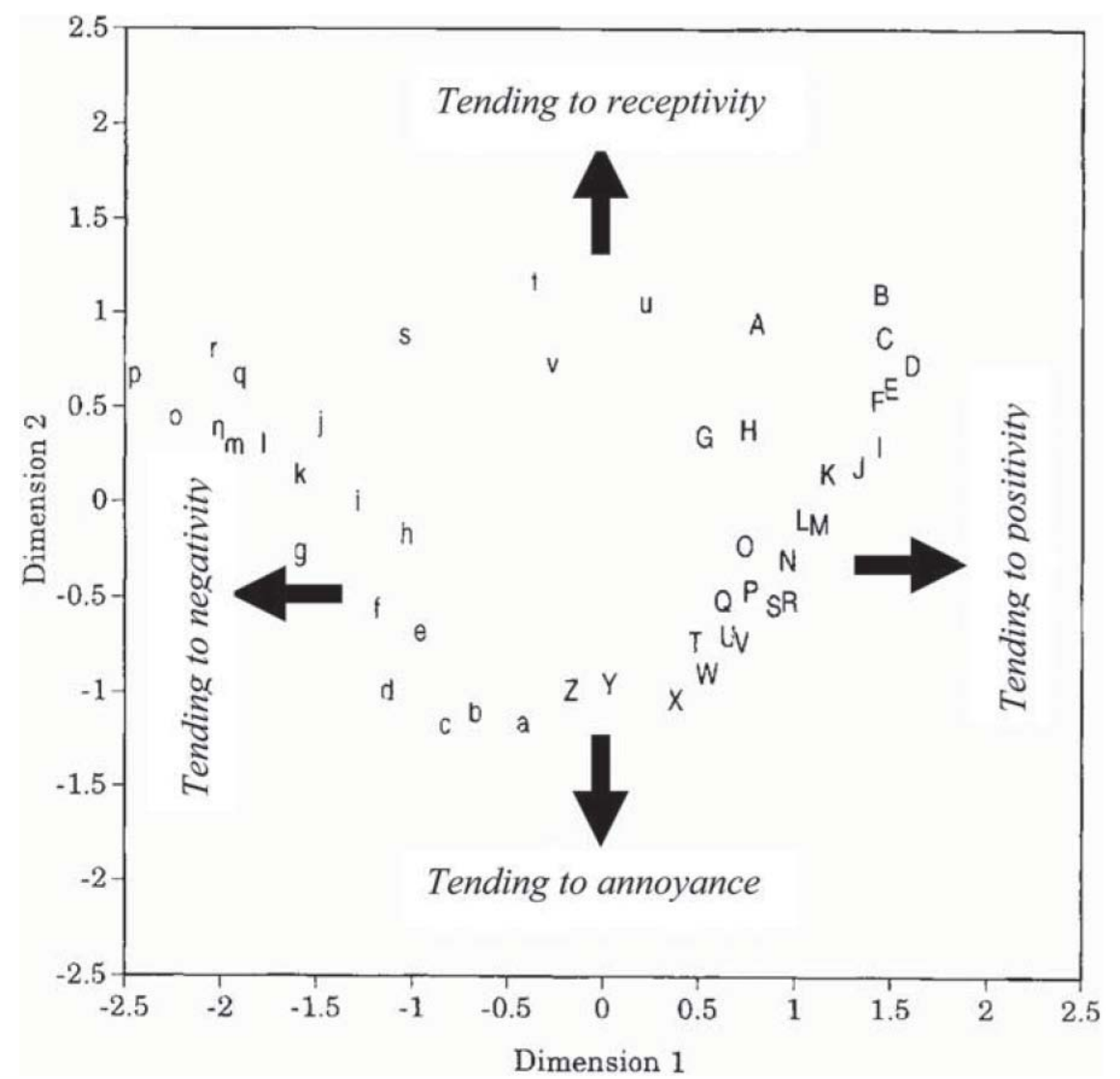

Figure 2 : Multi-dimension scaling of emotions related to consumption (after Richins, 1997). In clockwise direction from the top, the letters represent: A, impatient; B, frustrated; C, irritated; D, angry; E, unfulfi lled ; F, discontented ; G, worried; H, tense; I, disgusted; J, furious ; K, grouchy ; L, depressed ; M, miserable ; N, sad; $\mathrm{O}$, panicky; P, threatened; Q, afraid; R, ashamed; S, embarrassed; T, guilty ; U, envious; V, jealous ; W, lonely; X, homesick; Y, tender ; Z, sexy; a, romantic ; b, loving; c, sentimental; d, warm-hearted ; e, calm ; f, peaceful ; g, comforted ; h, relieved ; I, hopeful; j, optimistic; k, contented ; l, fulfi lled ; m, proud ; n, joyful; o, glad ; p, pleased; q, enthusiastic; r, excited; s, eager; t, amazed ; u, surprised; v, overwhelmed . 


\section{Tending to receptivity}

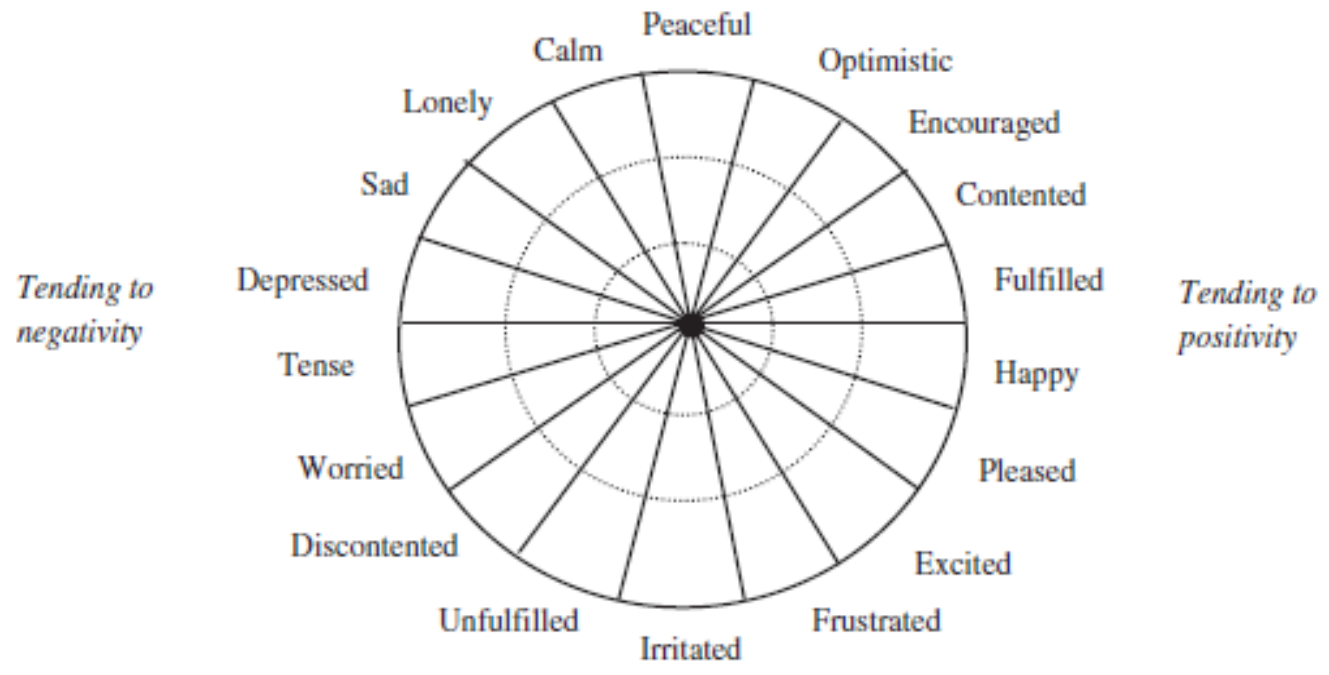

Tending to annoyance

Figure 3 : The emotions wheel used to collect the data.

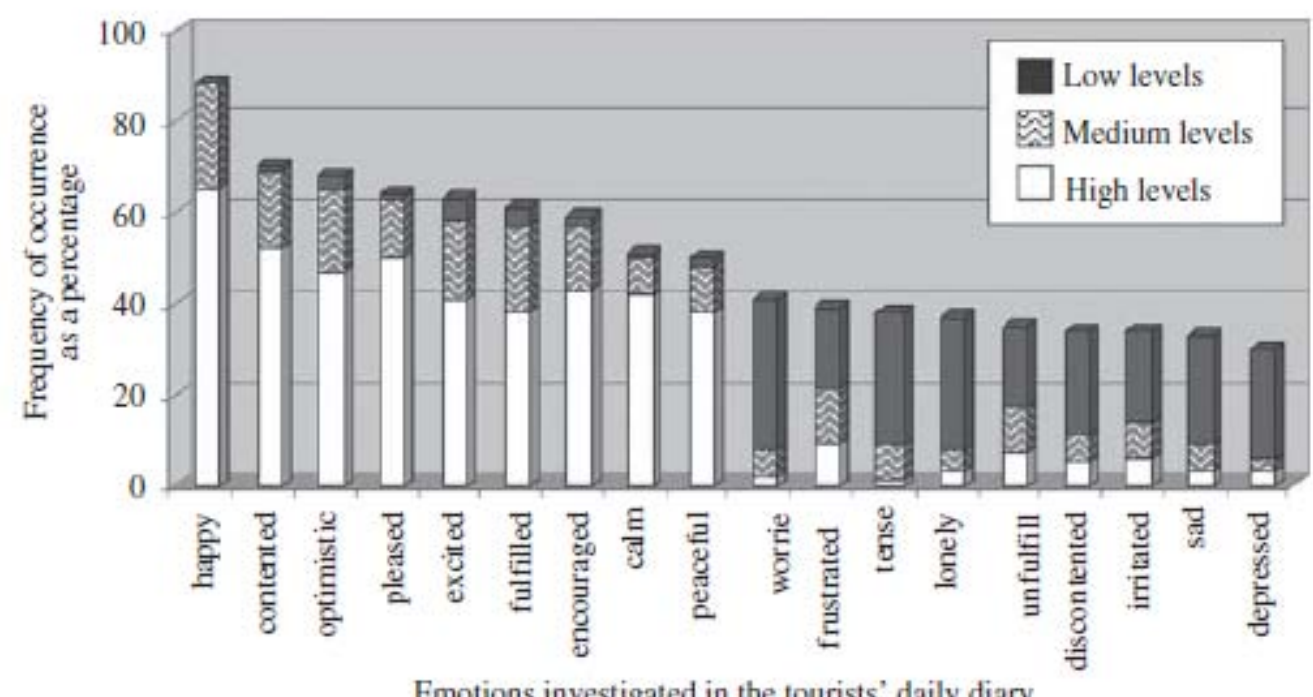

Figure 4 : Frequency (as a percentage) and intensity of emotions reported by the total volunteer tourism sample ( $N=16,10$ days in total). Darker shading represents a higher intensity of the emotion (that is emotional states reported in the outer segments of the wheel). 
Table 1 : The daily satisfaction and affect scores. Scores are given as an average for all respondents

\begin{tabular}{|c|c|c|c|c|c|}
\hline Day & $\begin{array}{c}\text { Satisfaction } \\
\text { score }\end{array}$ & Emotion type* recorded that day & Day & $\begin{array}{c}\text { Satisfaction } \\
\text { score }\end{array}$ & Emotion type* recorded that day \\
\hline 1 & 4.5 & $\begin{array}{l}\text { High: All positive emotions \& in } \\
\text { particular, calm, optimistic, content, } \\
\text { happy, pleased, excited. } \\
\text { Low: All negative emotions }\end{array}$ & 6 & 4.6 & $\begin{array}{l}\text { High: All positive emotions \& in } \\
\text { particular, optimistic, encouraged, } \\
\text { content, happy, pleased, Frustrated } \\
\text { Low: irritated discontented, worried, } \\
\text { tense, depressed, sad, lonely }\end{array}$ \\
\hline 2 & 4.3 & $\begin{array}{l}\text { High: All positive emotions \& in } \\
\text { particular, optimistic, happy, pleased, } \\
\text { excited. } \\
\text { Low: All negative emotions } \\
\text { High: All positive emotions \& in }\end{array}$ & 7 & 4.3 & $\begin{array}{l}\text { High: All positive emotions \& in } \\
\text { particular, calm, peaceful, optimistic, } \\
\text { fulfilled, happy, pleased. } \\
\text { Medium: Frustrated discontented } \\
\text { Low: Irritated, unfulfilled, worried, } \\
\text { tense, depressed, sad, lonely }\end{array}$ \\
\hline & & $\begin{array}{l}\text { particular, optimistic, encouraged, } \\
\text { content, happy, pleased. } \\
\text { Medium: discontented, worried, } \\
\text { lonely } \\
\text { Low: All negative emotions }\end{array}$ & 8 & 4.7 & $\begin{array}{l}\text { High: All positive emotions } \\
\text { Medium: Frustrated, unfulfilled, } \\
\text { depressed. } \\
\text { Low: Irritated discontented, worried, } \\
\text { tense, sad, lonely }\end{array}$ \\
\hline 4 & 4.4 & $\begin{array}{l}\text { High: All positive emotions \& in } \\
\text { particular, optimistic, encouraged, } \\
\text { content, happy, pleased, Irritated } \\
\text { Medium: Frustrated, unfulfilled } \\
\text { Low: Discontented, worried, tense, } \\
\text { depressed, lonely. }\end{array}$ & 9 & 4.3 & $\begin{array}{l}\text { High: All positive emotions \& in } \\
\text { particular, calm, happy. } \\
\text { Medium: Frustrated } \\
\text { Low: Irritated discontented, worried, } \\
\text { tense, depressed, sad, lonely }\end{array}$ \\
\hline 5 & 4.2 & $\begin{array}{l}\text { High: All positive emotions \& in } \\
\text { particular, calm, peaceful, content, } \\
\text { happy.. Frustrated } \\
\text { Medium: Irritated, unfulfilled } \\
\text { Low: Frustrated, discontented, } \\
\text { worried, tense, depressed, sad, lonely }\end{array}$ & 10 & 4.3 & $\begin{array}{l}\text { High: All positive emotions. } \\
\text { Medium: tense, sad, depressed } \\
\text { Low: Unfulfilled, discontented, } \\
\text { worried }\end{array}$ \\
\hline
\end{tabular}

*The emotion wheel was divided up into three segments based upon average distances between emotion in Richins's (1997) multiple dimensional scaling. A measure of distances revealed greater than average distances between lonely and calm $(4 \mathrm{~cm})$, excited and frustrated $(10.3 \mathrm{~cm})$ and discontented and worried $(3.5 \mathrm{~cm})$. Segments shaded in black represent average scores greater than one (that is, at least low levels of that emotion each day), segments shaded in dark grey represent average scores of $0.5-0.9$, while scores lower than 0.5 are represented in light grey. 


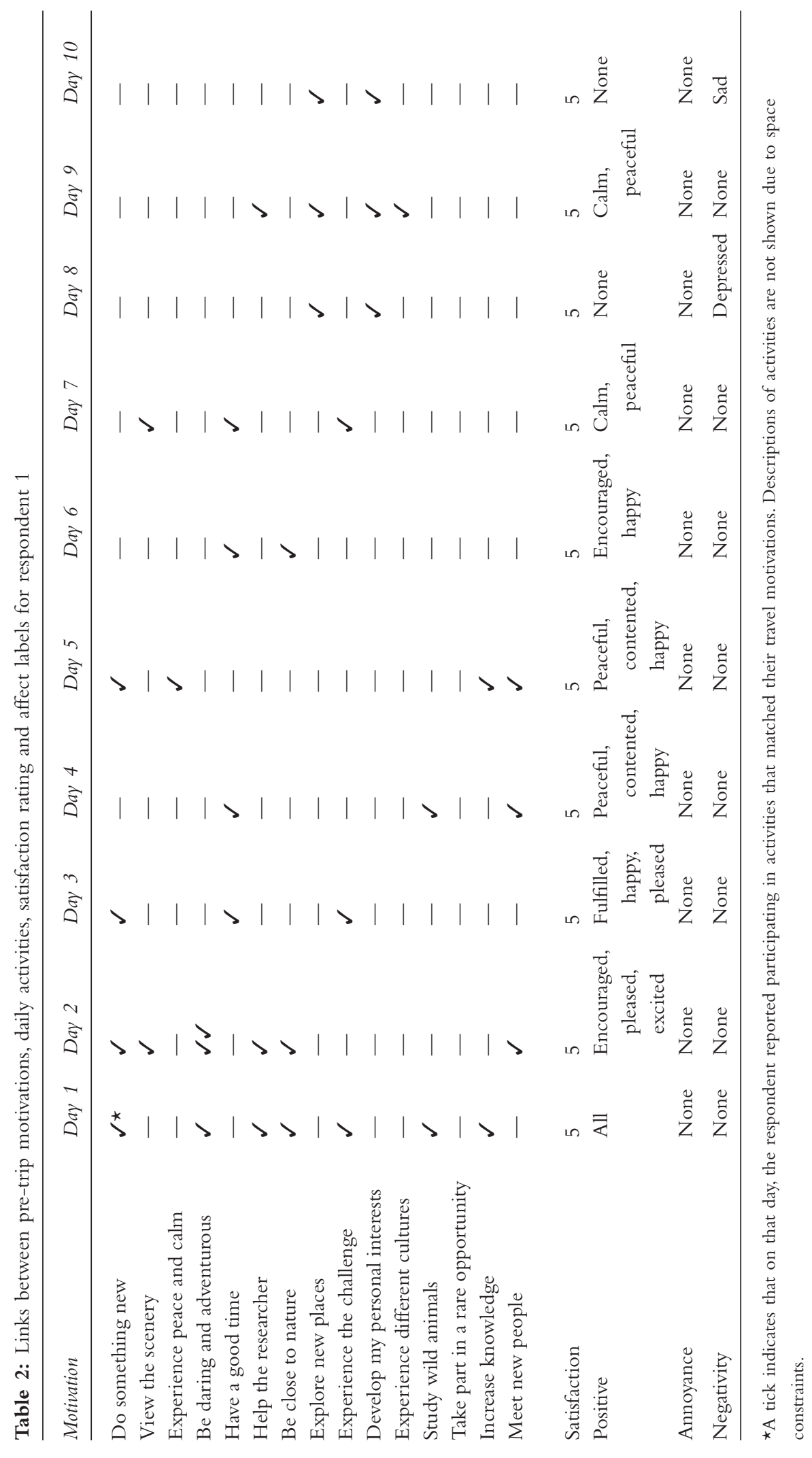




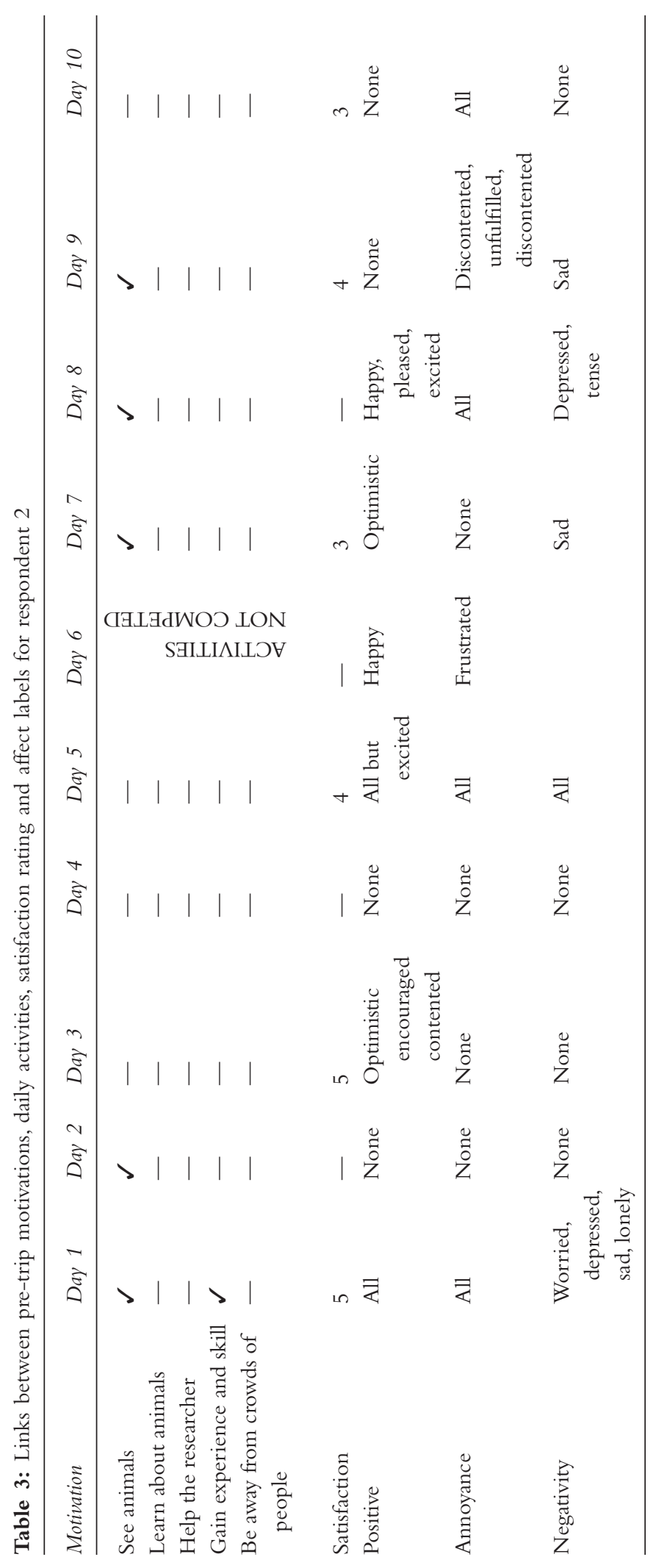




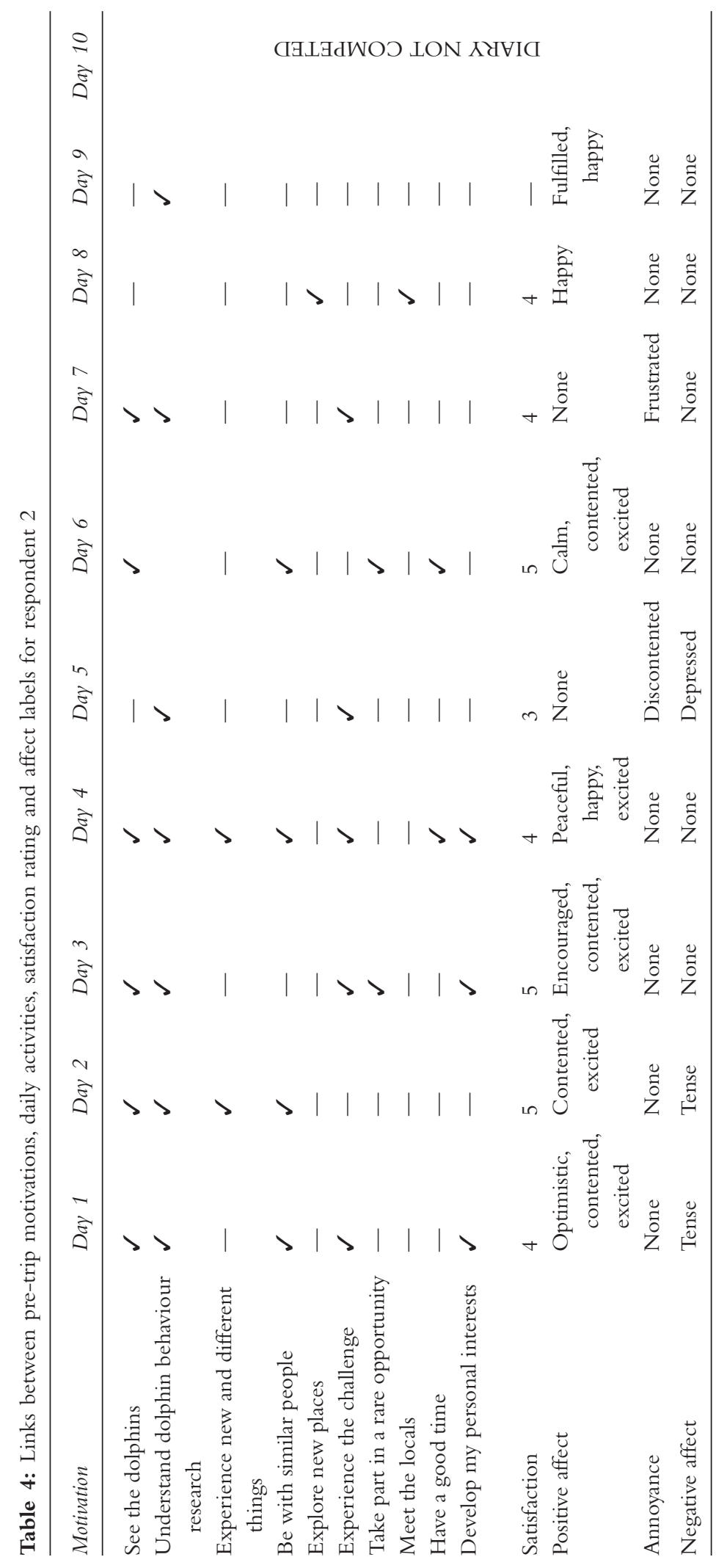




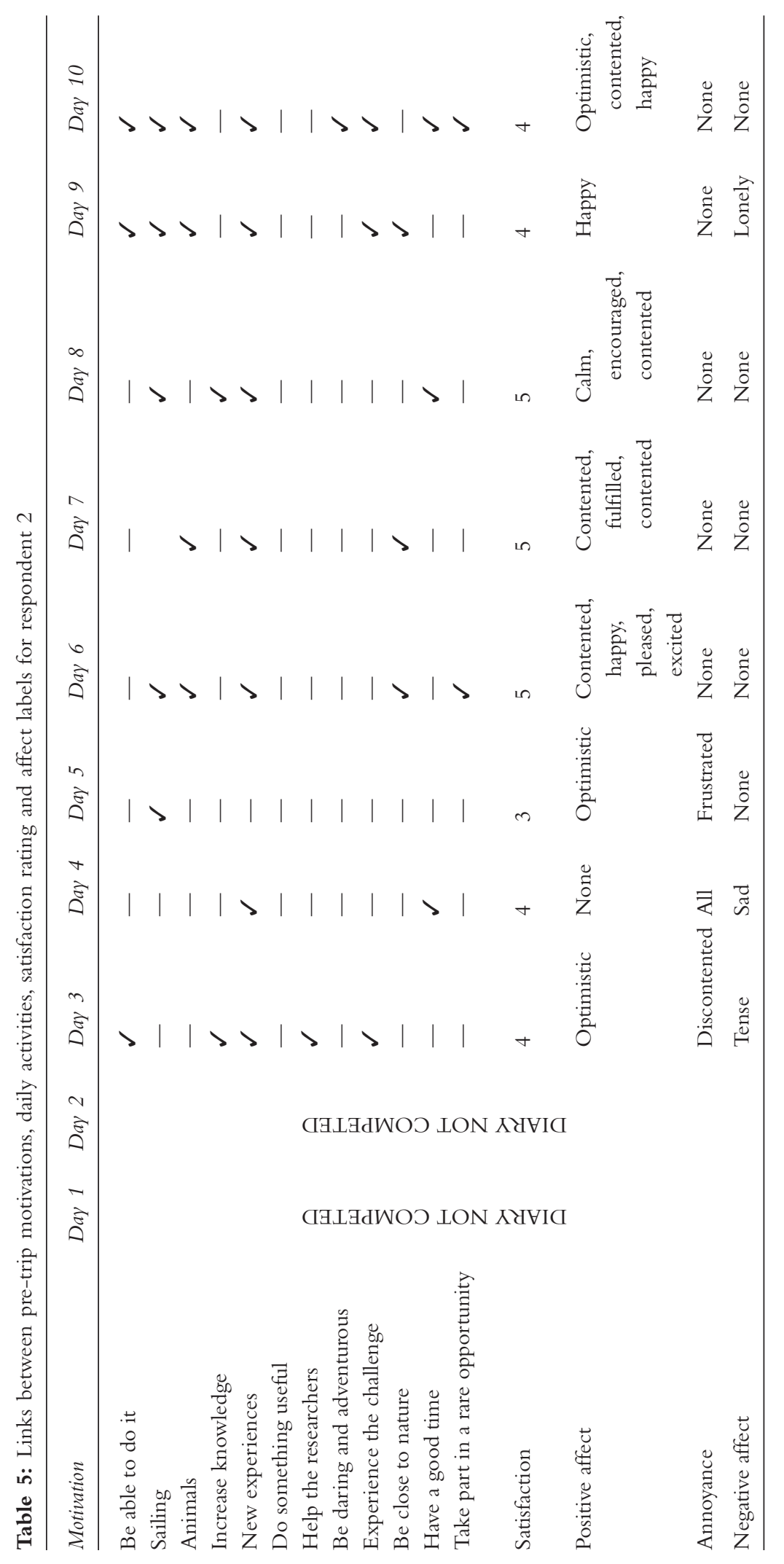

